ARTICLE

Received 31 Jul 2015 | Accepted 19 Feb 2016 | Published 30 Mar 2016

DOI: $10.1038 /$ ncomms11083

OPEN

\title{
Three-dimensional structural dynamics and fluctuations of DNA-nanogold conjugates by individual-particle electron tomography
}

Lei Zhang ${ }^{1,2}$, Dongsheng Lei ${ }^{1}$, Jessica M. Smith ${ }^{3,4,5}$, Meng Zhang ${ }^{1}$, Huimin Tong ${ }^{1}$, Xing Zhang ${ }^{1,2}$, Zhuoyang Lu' $1,6,7,8$, Jiankang Liu $6,7,8$, A. Paul Alivisatos ${ }^{3,4,5,9} \&$ Gang Ren ${ }^{1}$

DNA base pairing has been used for many years to direct the arrangement of inorganic nanocrystals into small groupings and arrays with tailored optical and electrical properties. The control of DNA-mediated assembly depends crucially on a better understanding of threedimensional structure of DNA-nanocrystal-hybridized building blocks. Existing techniques do not allow for structural determination of these flexible and heterogeneous samples. Here we report cryo-electron microscopy and negative-staining electron tomography approaches to image, and three-dimensionally reconstruct a single DNA-nanogold conjugate, an 84-bp double-stranded DNA with two 5-nm nanogold particles for potential substrates in plasmoncoupling experiments. By individual-particle electron tomography reconstruction, we obtain 14 density maps at $\sim 2$-nm resolution. Using these maps as constraints, we derive 14 conformations of dsDNA by molecular dynamics simulations. The conformational variation is consistent with that from liquid solution, suggesting that individual-particle electron tomography could be an expected approach to study DNA-assembling and flexible protein structure and dynamics.

\footnotetext{
${ }^{1}$ The Molecular Foundry, Lawrence Berkeley National Laboratory, Berkeley, California 94720, USA. ${ }^{2}$ Department of Applied Physics, School of Science, Xi'an Jiaotong University, Xi'an 710049, China. ${ }^{3}$ Materials Sciences Division, Lawrence Berkeley National Laboratory, Berkeley, California 94720, USA.

${ }^{4}$ Department of Chemistry, University of California, Berkeley, California 94720, USA. ${ }^{5}$ Department of Materials Science, University of California, Berkeley, California 94720, USA. ${ }^{6}$ Center for Mitochondrial Biology and Medicine, The Key Laboratory of Biomedical Information Engineering of the Ministry of Education, Xi'an Jiaotong University, Xi'an 710049, China. ${ }^{7}$ School of Life Science and Technology, Xi'an Jiaotong University, Xi'an 710049, China. ${ }^{8}$ Frontier Institute of Science and Technology, Xi'an Jiaotong University, Xi'an 710049, China. ${ }^{9}$ Kavli Energy NanoScience Institute, University of California, Berkeley, California 94720, USA. Correspondence and requests for materials should be addressed to G.R. (email: gren@lbl.gov).
} 
O rganic-inorganic-hybridized nanocrystals are a valuable class of new materials that are suitable for addressing many emerging challenges in biological and material sciences ${ }^{1,2}$. Nanogold and quantum dot conjugates have been used extensively as biomolecular markers ${ }^{3,4}$, whereas DNA base pairing has directed the self-assembly of discrete groupings and arrays of organic and inorganic nanocrystals in the formation of a network solid for electronic devices and memory components 5 . Discretely hybridized gold nanoparticles conjugated to DNA were developed as a molecular ruler to detect sub-nanometre distance changes via plasmon-coupling-mediated variations in dark-field light scattering ${ }^{3,6}$. For many of these applications, it is desirable to obtain nanocrystals functionalized with discrete numbers of DNA strands ${ }^{7,8}$. In all of these circumstances, the soft components can fluctuate, and the range of these structural deviations have not previously been determined with a degree of rigour that could help influence the future design and use of these assemblies.

Conformational flexibility and dynamics of the DNA-nanogold conjugates limit the structural determination by X-ray crystallography, nuclear magnetic resonance spectroscopy and singleparticle cryo-electron microscopic (cryo-EM) reconstruction because they do not crystallize, are not sufficiently small for nuclear magnetic resonance studies and cannot be classified into a limited number of classes for single-particle EM reconstruction. In addition, three-dimensional (3D) structure averaged from tens of thousands of different macromolecular particles obtained without prior knowledge of the macromolecular structural flexibility could result in an absence of flexible domains upon using the single-particle reconstruction method, for example, two ankyrin repeated regions of TRPV1 were absent in its atomic resolution 3D density map 9 .

A fundamental experimental solution to reveal the structure of a flexible macromolecule should be based on the determination of each individual macromolecule's structure ${ }^{10}$. Electron tomography (ET) provides high-resolution images of a single object from a series of tilted viewing angles ${ }^{11}$. ET has been applied to reveal the 3D structure of a cell section and an individual bacterium at nanometre-scale resolution ${ }^{12}$. However, reconstruction from an individual macromolecule at an intermediate resolution $(1-3 \mathrm{~nm})$ remains challenging due to small molecular weight and low image contrast. Although, the first 3D map of an individual macromolecule, a fatty acid synthetase molecule, was reconstructed from negative-staining (NS) ET by Hoppe et al. ${ }^{13}$, serious doubts have been raised regarding the validity of this structure ${ }^{14}$, as this molecule received a radiation dose hundreds of times greater than the reported damage threshold ${ }^{15}$. Recently, we investigated the possibility based on simulated and real experimental NS and cryo-ET images ${ }^{10}$. We showed that a single-protein $3 \mathrm{D}$ structure at an intermediate resolution $(1-3 \mathrm{~nm})$ is potentially achieved using our proposed individual-particle ET (IPET) method ${ }^{10,16-18}$. IPET, an iterative refinement process using automatically generated dynamic filters and soft masks, requires no pre-given initial model, class averaging or lattice, but can tolerate small tilt errors and large-scale image distortion via decreasing the reconstruction image size to reduce the negative effects on $3 \mathrm{D}$ reconstruction. IPET allows us to obtain a 'snapshot' singlemolecule 3D structure of flexible proteins at an intermediate resolution, and can be even used to reveal the macromolecular dynamics and fluctuation ${ }^{17}$.

Here we use IPET, cryo-EM and our previously reported optimized NS $(\mathrm{OpNS})^{19,20}$ techniques to investigate the morphology and 3D structure of hybridized DNA-nanogold conjugates. These conjugates were self-assembled from a mixture of two monoconjugates, each consisting of 84-bp single-stranded DNA and a 5-nm nanogold particle. The dimers were separated by anion-exchange high-performance liquid chromatography (HPLC) and agarose gel electrophoresis as potential substrates in plasmon-coupling experiments. By OpNS-ET imaging and IPET 3D reconstruction, we reconstruct a total of 14 density maps at a resolution of $\sim 2 \mathrm{~nm}$ from 14 individual doublestranded DNA (dsDNA)-nanogold conjugates. Using these maps as constraints, we derive 14 conformations of dsDNA by projecting a standard flexible dsDNA model onto the observed maps using molecular dynamics (MD) simulations. The variation of the conformations was largely consistent with that from liquid solution, and suggests that the IPET approach provides a most complete experimental determination of flexibility and fluctuation range of these directed nanocrystal assemblies to date. The general features revealed by this experiment can be expected to occur in a broad range of DNA-assembled nanostructures and flexible proteins.

\section{Results}

Cryo-EM and NS images of DNA-nanogold conjugates. The sample of HPLC-purified 84-bp dsDNA (molecular mass $=\sim 52 \mathrm{kDa}$ ) and two 5-nm nanogold conjugates (Supplementary Fig. 1) were examined by two methods, that is, cryo-EM (native buffer, vitreous ice, no staining) and OpNS ${ }^{19,20}$. Cryo-EM, a method often used to prevent artefacts induced by fixatives and stains, can be used to study protein structures under near-native conditions. However, small proteins $(<100 \mathrm{kDa})$ are challenging to be visualized or $3 \mathrm{D}$ reconstructed. NS, a historical method, can be used for high-contrast imaging of small proteins through heavy metal salts coating the proteins surfaces ${ }^{20,21}$ However, heavy metal reaction can also cause a potential artefact by conventional protocols, including rouleaux formation of lipoproteins ${ }^{19,22}$. We used cryo-EM as a control, investigated the parameters related to lipoprotein rouleaux artefact ${ }^{19}$ and reported an OpNS protocol ${ }^{19,22}$. The OpNS protocol has been tested by structure-known proteins, including $53-\mathrm{kDa}$ cholesteryl ester transfer protein ${ }^{23}$, GroEL and proteasomes ${ }^{20}$, and the flexible proteins with structure partially known, including immunoglobulin (Ig)-G1 antibody and its peptide conjugates ${ }^{16,17}$. The surrounding heavy metal atoms provide more electron scattering and radiation damage resistance than the protein light atoms. In this study, we used cryo-EM and OpNS methods to examine the same sample under $-178^{\circ} \mathrm{C}$ and room temperature, respectively, (Fig. 1).

Survey cryo-EM and OpNS-EM micrographs (Fig. 1a,e) showed that each pair of nanogold particles was near one another. A statistical analysis of 1,032 nanogold particles from cryo-EM showed that the particles had a diameter of $\sim 63.5 \pm 6.7 \AA$ (mean \pm s.d.) and a peak population $(\sim 25.1 \%)$ diameter of $63.6 \pm 1.0 \AA$ (black solid line in Fig. 1c). This measurement is consistent with those from OpNS, that is, 606 nanogold particles from NS micrographs showed a diameter of $\sim 63.0 \pm 6.4 \AA$ (mean \pm s.d.) and a peak population $(\sim 25.5 \%)$ diameter of $62.8 \pm 1.0 \AA$ (blue dashed line in Fig. 1c).

To quantitatively identify whether the pairs of nanogold particles are strongly linked together by a statistical method, Pearson's correlation coefficients (PCC) ${ }^{24}$ were used. The PCC, $r_{x, x}$ (defined as $r_{x, x}=\sum_{i=1}^{n}\left(x_{i}^{a}-\bar{x}^{a}\right)\left(x_{i}^{b}-\bar{x}^{b}\right) / \sqrt{\sum_{i=1}^{n}\left(x_{i}^{a}-\bar{x}^{a}\right)^{2} \sum_{i=1}^{n}\left(x_{i}^{b}-\bar{x}^{b}\right)^{2}}$ for the $x$ axis coordinates of two objects, $a$ and $b$ ) and $r_{y, y}$ for the $y$ axis coordinates were 0.9996 and 0.9976 for cryo-EM, and 0.9984 and 0.9983 for NS-EM results, respectively. The coefficients corresponded well with previous transmission electron microscopy (TEM) observations of the same sample in liquid solution (that is, $r_{x, x}=0.934$ and $r_{y, y}=0.943$; ref. 24). The high PCC suggests that the pair of nanogold particles are strongly linked together ${ }^{24}$. 


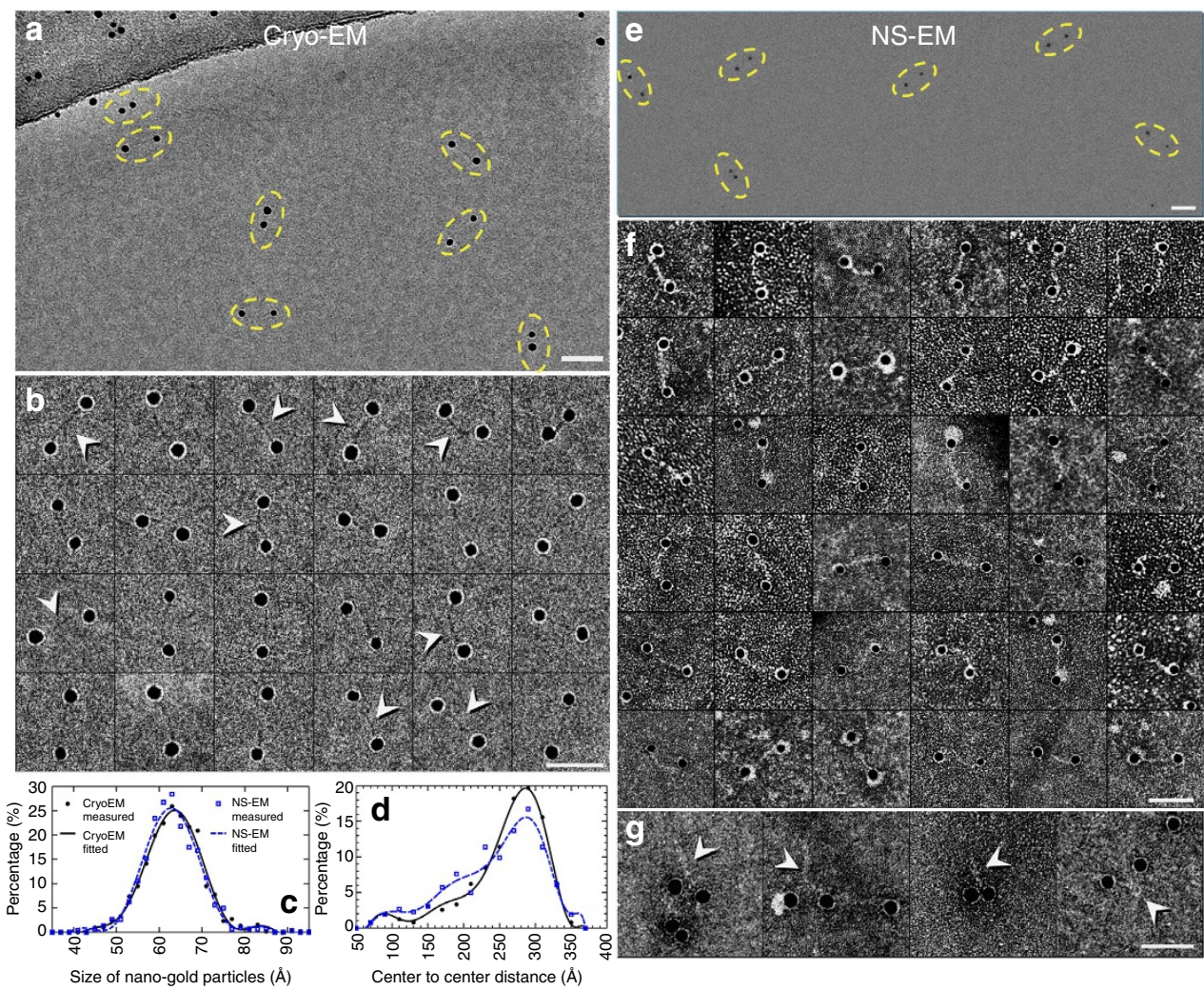

Figure 1 | Cryo-EM and OpNS-EM images of dsDNA-nanogold conjugates. (a) Cryo-EM images (vitreous buffer, no staining) of 5-nm nanogold particles conjugated to 84-bp dsDNA via a 5'-thiol linker. Pairs of nanogold were marked by yellow dashed ovals. (b) 24 representative cryo-EM images of the particles of DNA-nanogold conjugates. The polygonal-shaped areas are the nanogold particles, which were bridged by a fibre-shaped density (high contrast densities were indicated by arrows), $\sim 20-30 \mathrm{~nm}$ in length and $\sim 2 \mathrm{~nm}$ in width. The surfaces of the nanogold particles were coated with a layer of extraneous PEG for surface protection. (c) Histogram of the geometric diameters of 1,032 nanogold particles from cryo-EM images and 606 nanogold particles from NS images. (d) Histogram of the DNA lengths measured from 516 conjugates from cryo-EM and 303 conjugates from NS. The centre-to-centre length was measured between the centres of each nanogold particle pair. (e) NS images and (f) 36 representative NS images of the particles. The polygonal-shaped nanogold particles were bridged by a fibre-shaped density, and their surfaces were coated with a layer of extraneous PEG for surface protection. ( $(\mathbf{g}$ ) A few pairs of nanogold particles were significantly closer in distance to each other, whereas their bridging fabric-like densities were thicker (indicated by arrows), likely due to the supercoiling of the dsDNA. Scale bars $=30 \mathrm{~nm}$.

Zoom-in images of 24 representative cryo-EM particle pairs (Fig. 1b) and 36 representative NS particle pairs (Fig. 1f) showed that the polygonal-shaped nanogold particles were bridged by an $\sim 2$-nm-width fibre-shaped density. A statistical analysis of the distances among the 516 pairs of cryo-EM nanogold particles yielded a length of $255.3 \pm 48.7 \AA$ (mean \pm s.d., measured from the centre to centre of the nanogold particles) with a peak population $(\sim 19.7 \%)$ distance of $286.4 \pm 10 \AA$ (black solid line in Fig. 1d). The distances are similar to that from 303 pairs of NS particles, that is, $245.5 \pm 62.6 \AA$ (mean \pm s.d.) with a peak population $(\sim 15.6 \%)$ distance of $287.0 \pm 10 \AA$ (blue dashed line in Fig. 1d). Moreover, both lengths were consistent with those measured from liquid solution by small-angle X-ray scattering (SAXS), that is, $28-30 \mathrm{~nm}$ (ref. 25) and a standard model of 84-bp dsDNA ( $\sim 2 \mathrm{~nm}$ wide and $\sim 30 \mathrm{~nm}$ long).

In addition, several pairs of nanogold particles presented abnormally closer to one another in both cryo-EM and NS. The higher-contrast NS images showed that their fibre-shaped bridging densities appeared thicker, but had lengths ranging from $\sim 20$ to $30 \mathrm{~nm}$, thus seeming similar to those of the fulllength dsDNA (Fig. 1g). We suspected that these particles may be formed by two conjugates, in which each conjugate lost one nanogold, but met and formed a supercoil via their two dsDNAs. Since the mass is only $52 \mathrm{kDa}$ above that of the regular conjugates, these particles are too small to be identified or isolated by our filtration.
3D reconstruction of an individual dsDNA-nanogold conjugate. To obtain a 3D structure of the dsDNA-nanogold conjugates, we used the IPET technique ${ }^{10}$ rather than the conventional singleparticle reconstruction method or sub-volume averaging method because these conjugates were not guaranteed to share the same structure (DNA is naturally flexible and dynamic in structure). IPET is used to obtain the $a b$ initio 3D structure of an individual particle imaged from a series of tilt angles (Fig. 2a).

Although the DNA portion in the cryo-EM images could be barely visible under a dose of $\sim 20 \mathrm{e}^{-} \AA^{-2}$, beyond this dose limitation, the contrasts rapidly disappeared, which prevents us from a full ET data acquisition ( $\sim 80$ micrographs $)$ and further $3 \mathrm{D}$ reconstruction. Thus, we used OpNS-EM for $3 \mathrm{D}$ reconstruction.

Although the signal-to-noise ratios (SNRs) of the nanogold portion of ET images (from $-60^{\circ}$ to $+60^{\circ}$ at $1.5^{\circ}$ increments) were only $\sim 0.19$ to $\sim 0.41$ with an average of $\sim 0.31$, the overall shape of dsDNA was still visible (Fig. $2 b$ and Supplementary Movie 1). After contrast transfer function (CTF) correction, the tilt images were iteratively aligned to a global centre before achieving a final $a b$ initio $3 \mathrm{D}$ reconstruction (Fig. $2 \mathrm{~b}$ ). During the iterations, SNR of the dsDNA portion gradually increased up to $\sim 2.44$. The final 3D showed an overall handcuff shape (Fig. 2c) at a resolution of $\sim 14.7 \AA$, measured based on a Fourier shell correlation (FSC) analysis (details are provided in the Methods section; black line in Fig. 2e). To avoid the potential 
a

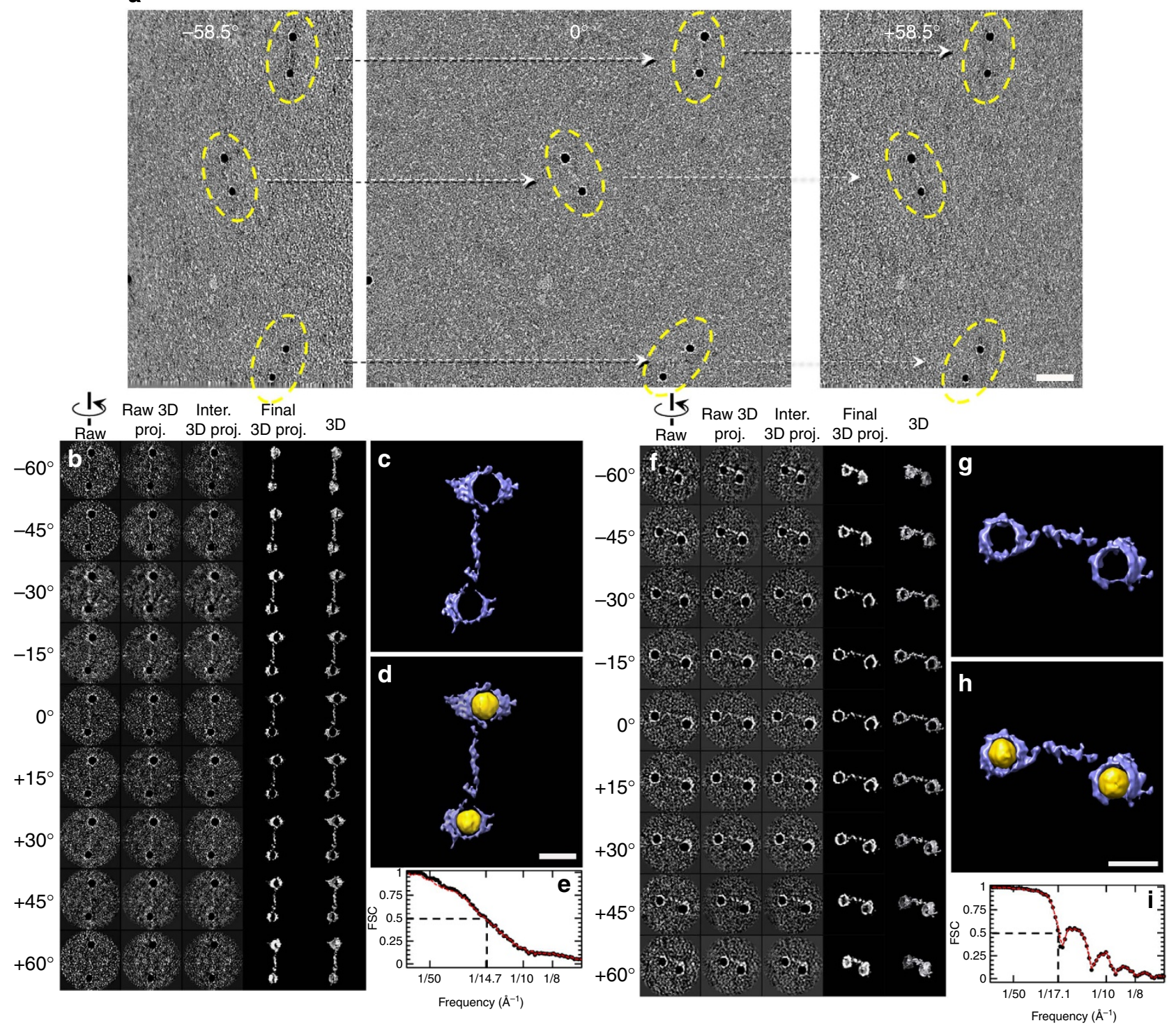

Figure 2 | 3D reconstruction of two representative DNA-nanogold conjugates by IPET. (a) The OpNS samples of DNA-nanogold conjugates were imaged using ET from a series of tilt angles (from $-60^{\circ}$ to $+60^{\circ}$ at $1.5^{\circ}$ intervals). Three targeted particles (yellow circled) with their orthogonal views are indicated by the linked dashed arrows in the three selected ET tilt micrographs. The relative tilt angles are indicated in each image, and the axis of the tilt is vertical to the images. (b) Nine representative tilt images of the first targeted individual particle are displayed in the first column from the left (SNR of DNA portion: 0.31). Using IPET, the tilt images (after CTF correction) were gradually aligned to a common centre for 3D reconstruction via an iterative refinement process. The projections of the intermediate and final 3D reconstructions at the corresponding tilt angles are displayed in the next four columns according to their corresponding tilt angles. (c) Final IPET 3D density map of the targeted individual particle (SNR of DNA portion: 2.44). (d) The final 3D density map and its overlaid 3D density maps (final map in blue and its reversed map in gold) indicated the overall conformation of the DNA-nanogold conjugates. (e) The FSC analyses under including (black line) and excluding (red line) nanogold portions (two density maps reconstructed from odd and even numbers of tilt images) revealed that the resolutions of the IPET 3D density map were both $\sim 14.7 \AA$. (f-i) The 3D density map of a second individual DNA-nanogold conjugate was reconstructed from the tilt images (SNR of DNA portion: 0.56) using IPET. The FSC analysis showed that the 3D reconstruction resolution (SNR of DNA portion: $\sim 3.26$ ) was $\sim 17.1 \AA$. Scale bars $=20 \mathrm{~nm}$ (a) and $10 \mathrm{~nm}(\mathbf{d}, \mathbf{h})$.

overestimation of resolution by nanogolds instead of correctly reflecting the resolution of the dsDNA, we masked out the dsDNA portion only to repeat the FSC analyses. The FSC curve was nearly identical to that for nanogolds (red versus black lines in Fig. 2e), suggesting that the resolution is not overestimated by nanogolds.

The nanogold particle surface appeared with a layer of densities, which is possibly thiolated short-chain polyethylene glycol (PEG) molecules used to stabilize the particles against aggregation at high ionic strength (Fig. 2c). Considering that the nanogold particles were in opposite image contrast to the dsDNA, we reversed the image contrast of the final $3 \mathrm{D}$ (coloured in gold) and overlaid it with original 3D to display both the dsDNA and nanogold particles in a same 3D map (Fig. 2d). This overlaid map showed the nanogold particles with diameters of $\sim 73.0$ and $\sim 72.0 \AA$ bridged by a high-density fabric with dimensions of $\sim 242.0 \AA \times \sim 18.0 \AA \times$ $\sim 18.0 \AA$ (Fig. 2d). The nanogold particle surfaces were surrounded by irregularly shaped densities, the PEG surface protection layer. 
Although the $3 \mathrm{D}$ resolution was insufficient to determine the structure of the dsDNA, the overall shape could be used as a constraint to flexibly dock a standard structure of an 84-bp dsDNA into it to achieve a dsDNA conformation. By satisfying both the best fit to the density map and the chemical minimal energy requirements, we obtained a dsDNA conformation via gently bending the straight dsDNA structure into the density map using the CHARMM force field for all MD simulations ${ }^{26,27}$ (Fig. 3a and Supplementary Movie 1).

By repeating the above process, we reconstructed $3 \mathrm{D}$ from second individual dsDNA-nanogold conjugate (Fig. 2f-i). The representative tilt images showed that the dsDNA was still visible (Fig. $2 \mathrm{f}$ ), and the SNR of dsDNA portions ranged from $\sim 0.41$ to $\sim 0.62$, with an average of 0.56 . Through IPET reconstruction, the tilt images were aligned to a global centre (Fig. 2f), and the SNR was increased up to $\sim 3.26$ (Fig. $2 \mathrm{~g}$ ). The final $3 \mathrm{D}$ reconstruction at $\sim 17.1-\AA$ resolution (based on the FSC analyses with and without the nanogold portion conditions; Fig. 2i) showed a handcuff shape. The overlaid density map (the final 3D map with its reversed map, coloured in gold) showed that the nanogold particles had diameters of $\sim 71.0$ and $\sim 65.0 \AA$ bridged by a fabric-like density with dimensions of $\sim 191.0 \AA \times$ $\sim 20.0 \AA \times \sim 20.0 \AA$ (Fig. 2h). Again, the nanogold particles were surrounded by irregularly shaped densities of a PEG surface protection layer. By flexibly docking the standard dsDNA structure into the bridging portion and following MD simulation for energy minimization, a second conformation of dsDNA was obtained (Fig. 3b).

3D reconstructions of another 12 DNA-nanogold conjugates. Through particle-by-particle 3D reconstructions, additional 12 conjugates were reconstructed using IPET (Fig. 3, Supplementary Figs 2-13 and Supplementary Table 1). The selected projections of the final $3 \mathrm{D}$ reconstructions showed a fabric DNA density between two nanogold particles (Fig. 3c). The overlaid density
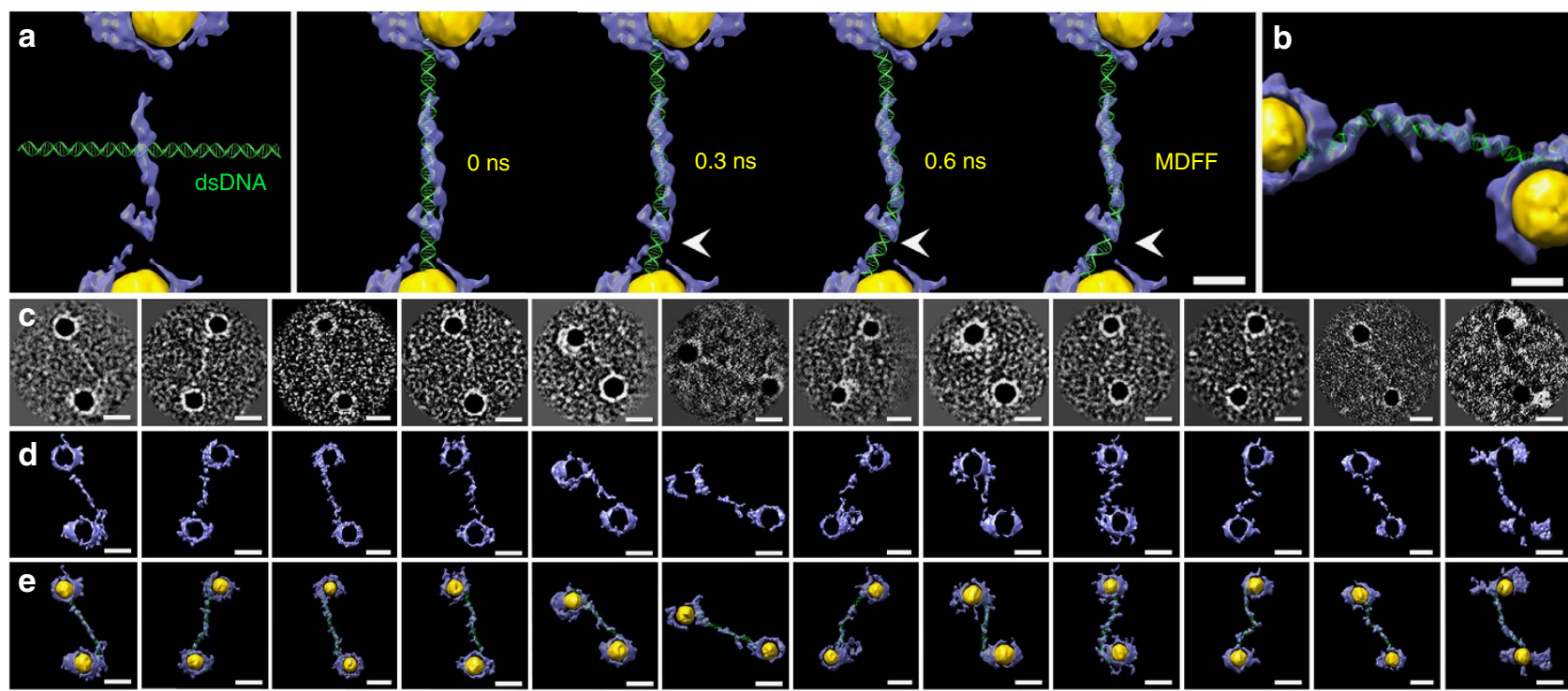

$\mathbf{f}$
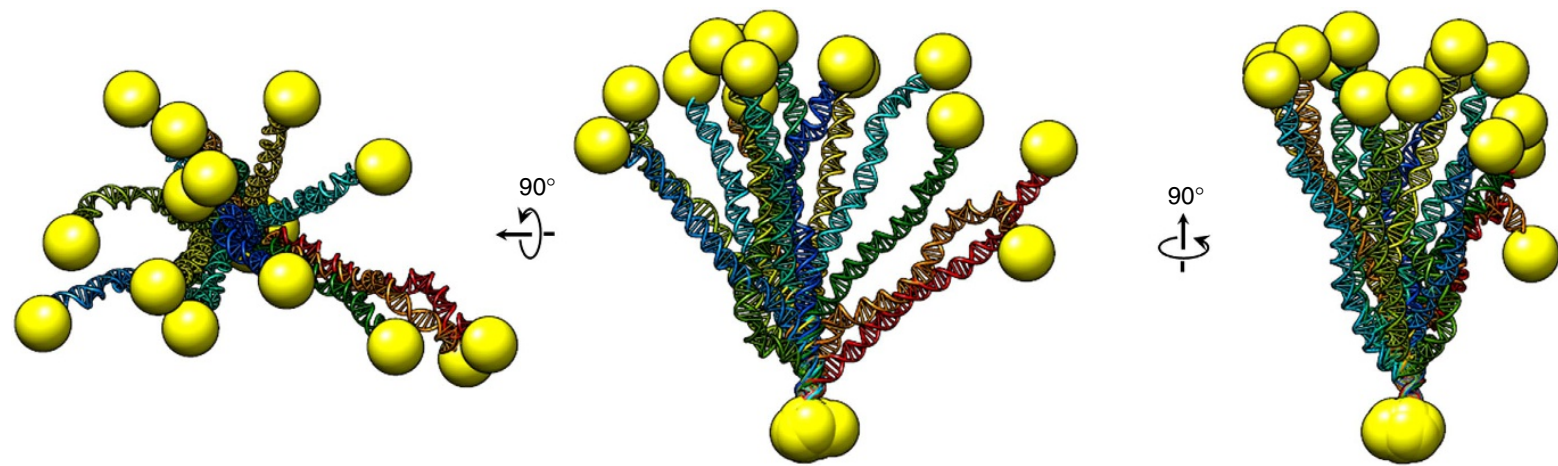

Figure 3 | 3D conformations of 14 dsDNA structures. Conformations obtained by flexibly fitting the dsDNA model onto the EM density maps using targeted MD simulations. (a) The final density map provided a constraint for the TMD simulations to achieve a new DNA conformation. Four snapshot images during the TMD simulation illustrate the process of flexibly docking the DNA model into the IPET density map to achieve a new conformation of DNA (arrow indicated the great conformational changing portion of DNA). During this process, the DNA conformation was allowed to change its structure while maintaining its chemical geometry and bonds with local energy minimization. (b) The final conformation of the second dsDNA structure was obtained from the second density map by following the same processes. (c-e) Gallery of 12 additional conformations from the 3D density maps of an additional 12 DNA-nanogold conjugates reconstructed using IPET. (c) Selected projections of the 3D density map of each individual DNA-nanogold conjugate. (d) Final 3D density maps of the individual DNA-nanogold conjugates. (e) The overlaid density maps of the final 3D reconstruction (grey) and its reversed contrast map (gold) revealed the overall conformation of the DNA-nanogold conjugates. A standard 84-bp dsDNA structure was flexibly docked into each density map to achieve the new conformations via TMD simulations. (f) Conformational flexibility and dynamics of the DNA-nanogold conjugate. Fourteen conformations of the DNA-nanogold conjugates were aligned together based on their first 14 bp. The distribution of dsDNA is shown from three orthogonal views. Scale bars $=5 \mathrm{~nm}(\mathbf{a}, \mathbf{b})$ and $10 \mathrm{~nm}(\mathbf{c}-\mathbf{e})$. 
maps (reversed maps are coloured in gold; Fig. 3d,e) also confirmed that the nanogold particles were connected by densities attributable to dsDNA. Although up to $\sim 30 \%$ of the fabric-like densities in those maps (Fig. 3e) could not be fully observed under the selected contour levels owing to various factors, such as uneven staining, image noise and reconstruction errors, these defects have a limited effect on the spacing distribution and the overall shape determination of the dsDNA due to its connectivity. The resolutions of $\sim 20 \AA$ still allowed us to flexibly dock the dsDNA model to obtain 12 additional DNA conformations via MD simulations (Fig. 3e and Supplementary Figs 2c-13c).

Statistical analyses of DNA-nanogold conjugates structures. Aligning the 14 conformations of dsDNA along their first $14 \mathrm{bp}$ yielded a distribution in a shape of a bundle of flowers (Fig. 3f). Considering that the 14 conformations is insufficient to reveal the full 3D distribution of DNA conformations, only 1D distribution analysis was conducted, that is, the nanoparticle size and DNA length. The histogram of the nanogold particle sizes measured from the $3 \mathrm{D}$ reconstructions (the measurement method is described in Methods section) showed that the geometric mean of the nanoparticle diameters was $65.7 \pm 5.0 \AA$, which is similar to the diameter measured from the $2 \mathrm{D}$ images of 606 nanogold particles $(62.8 \pm 1.0 \AA$ at peak population, $\sim 25.5 \%$; Fig. $1 \mathrm{~d}$ and Supplementary Table 1). The average distance measured from the $3 \mathrm{D}$ reconstructions (the measurement method is described in Methods section) was $291.1 \pm 31.9 \AA$ (mean \pm s.d.), which was longer than the mean distance measured from the $2 \mathrm{D}$ images $(245.5 \pm 62.6 \AA)$, but was similar to the distance of $287.0 \pm 10.0 \AA$ at the peak population $(\sim 15.6 \%$; Fig. 1 d). Approximately $69 \%$ of the distances was shorter than the peak distance, whereas $\sim 31 \%$ were longer (Supplementary Table 1). This uneven distribution of the distance around the distance of the peak population is likely owing to the portion of dsDNA that formed a supercoiled structure but were not $3 \mathrm{D}$ reconstructed or measured. These variations reflected the conformational flexibility and dynamics of the dsDNA between the two conjugated nanogold particles.

Although the particles were flash-fixed by stain heavy metals and attached to a substrate (carbon film, which may cause certain artefacts, such as a preferred orientation, flatness and an uneven staining distribution), the statistical analyses showed that the measured lengths from 303 dimers were highly similar to the same sample measured in solution (Supplementary Table 2; refs 24,25). In detail, the distances measured using SAXS from the same sample in solution were $\sim 280 \AA$ on average and $\sim 320 \AA$ at the peak population ${ }^{25}$, which were $\sim 10-15 \%$ longer than those measured from our 2D images (Fig. 1d). Considering that the length measured from $2 \mathrm{D}$ images corresponded to the projection distance of the $3 \mathrm{D}$ length in solution, the $2 \mathrm{D}$ projection is naturally shorter than the length in $3 \mathrm{D}$ by a factor of $\pi / 4$ under an isotropic distribution assumption condition. Based on the solution measured distances, their corresponding $2 \mathrm{D}$ projection distances should be $\sim 220 \AA(\pi / 4 \times 280 \AA)$ on average and $\sim 250 \AA(\pi / 4 \times 320 \AA)$ at the peak population, which were $\sim 10-12 \%$ shorter than our measurement from $2 \mathrm{D}$ images. Our measured lengths $(\sim 246$ and $\sim 287 \AA)$ were between the 3D lengths $(\sim 280$ and $\sim 320 \AA)$ and the $2 \mathrm{D}$ projection lengths ( $\sim 220$ and $\sim 250 \AA)$, suggesting that our particles have a certain preferred orientation to the carbon film. The mean distance measured in solution via in situ TEM was $\sim 180 \AA$ (ref. 24), which was $\sim 26 \%$ shorter than the mean measured from our $2 \mathrm{D}$ images. Although the short-distance views of the targeted conjugate was specifically chosen for easy tracking and imaging in the in situ experiment, the mean value was still close to the error bar range in our measurement.
Analyses of bending energy of DNA-nanogold conjugates. The conformations of dsDNA provide an opportunity to study the DNA bending energy. The bending energy can be calculated based on a simple worm-like chain (WLC) model ${ }^{25,28,29}$, in which the energy of dsDNA is simplified to the bending potential while ignoring the distortion potential at the single-base pair level. The calculation of the bending energy depends on the local bending angles of each base pair, which is governed by a single parameter for the mechanical response, that is, the persistence length. Although the persistence length could be extracted by measuring the tangent correlation function for a WLC, the length of our 84-bp DNA here is too short to derive a persistence length. Thus, the widely used persistence length of $50 \mathrm{~nm}$ was used to compute the bending energy as this length is a favourable parameter to describe the dsDNA conformational statistics for constructs composed of tens of base pairs ${ }^{25}$.

To measure the local bending angle (Fig. 4a), each of two nearby base pairs was first fitted with a small standard cylinder with a fixed length and width (Fig. 4b,c). Two types of angles can be defined to represent the bending angle of the base pair, that is, the angle $\theta_{i}$, formed between centre-to-centre directions of three nearby cylinders (Fig. 4d), and the angle $\varphi_{i}$, formed between the two axes of nearby cylinders (Fig. 4e). The energies of each base pair were calculated and summed to represent the total energy of this dsDNA conformation. The energy distribution of the 14 conformations showed that the averaged bending energies were $\sim 116$ and $\sim 169 \mathrm{kcal} \mathrm{mol}^{-1}$ based on two types of angle definition (Fig. $4 \mathrm{f}$ and Supplementary Table 2). The average bending energies were $\sim 2-3$ times higher than the bending energy calculated based on a theoretical WLC model prediction on 84-bp DNA $\left(\sim 50 \mathrm{kcal} \mathrm{mol}^{-1} \text { at room temperature }\right)^{25}$, suggesting that the 14 DNA conformations were more flexible than the prediction.

Parameters influence the length and bending energy of DNA. Above analyses showed two definition types of bending angles, that is, $\theta_{i}$ and $\varphi_{i}$, which could cause a $\sim 50 \%$ difference on the computed bending energy from the experimental DNA conformations. Considering that the EM observed DNA is more flexible than predicted, it is worthy to evaluate whether other factors may also influence the measurement of the DNA length and bending energy, such as the fluctuation of distal ends, DNA modelling methods, noise bias in the EM density map, initial model bias and temperature. Additional analyses were performed as the following: (i) the bending energy based on the central $42 \mathrm{bp}$ was recalculated. This was because 84-bp DNA is relatively short and the ends of the chain exhibit greater fluctuations than the middle portion. The results obtained from the calculation showed that the bending energy is $\sim 15 \%$ less than when using all $84 \mathrm{bp}$ (Supplementary Table 2), suggesting that DNA is still more flexible than the WLC prediction. (ii) The initial model of DNA was obtained by manually fitting a standard DNA model to each EM configuration. Considering that the manual operation may lead to kinks, which are difficult to be repaired by MD simulation, we used another method to generate a smooth curved model of DNA whose structure was as close as possible to the canonical B-form DNA double helix (details are in Methods section). Before conducting any simulation, we calculated its bending energy, which is only $\sim 10 \%$ of the above bending energy, and only $\sim 20 \%$ of the WLC prediction, suggesting that the smooth DNA is more stiff than the WLC prediction, and EM configurations. (iii) After submitting this smooth model for energy minimization using the nanoscale molecular dynamics version 2 (NAMD2) software package ${ }^{30}$, we found that the bending energy was increased $\sim 4-5$ times, thus nearly approaching the WLC 
a

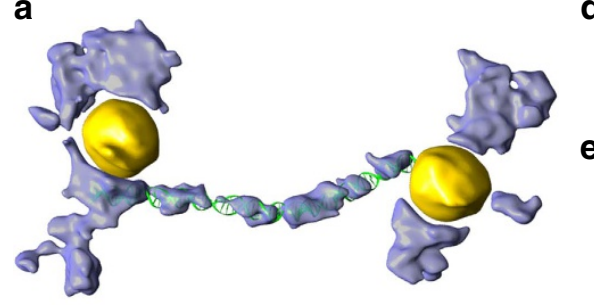

b

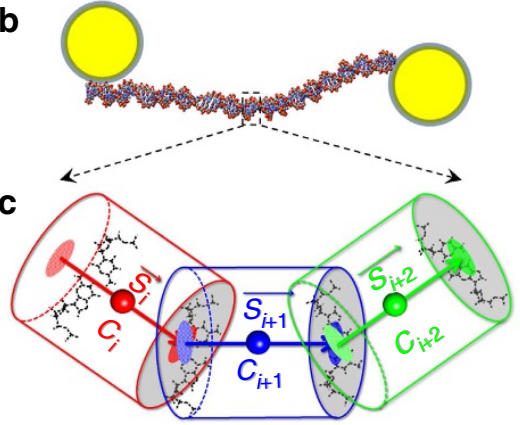

d

f e
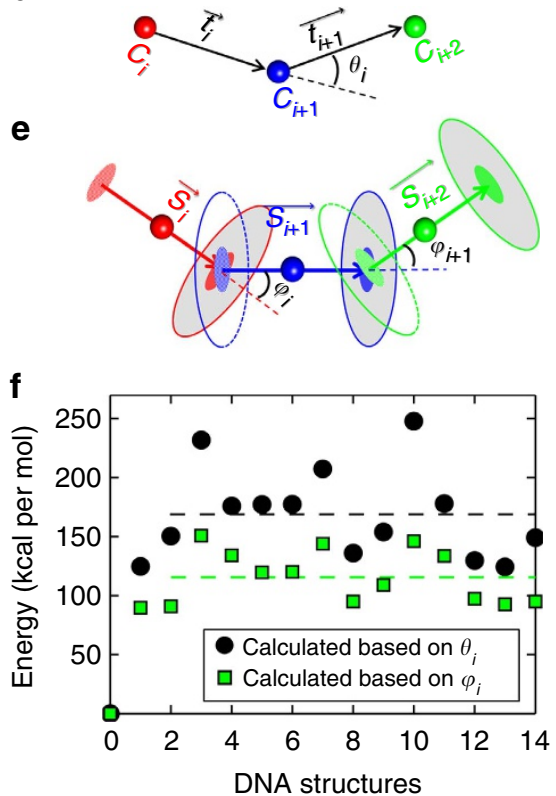

Figure 4 | Bending energy distribution of dsDNA. (a) dsDNA conformation was obtained by fitting the standard dsDNA model into the IPET 3D density map. (b) Schematic model illustrating that the nanogold interacts with the dsDNA and that the dsDNA contains kink regions that carry bending elasticity. (c) Cylinder model illustrating the bending angles between two connected base pairs. The cylinder is defined by the two consecutive dsDNA base pairs. (d) The bending angle can be presented by the angle $\theta_{i}$ formed by the centres of three consecutive cylinders, or (e) by the angle $\varphi_{i}$, formed by the centre axes of two consecutive cylinders. (f) Based on the two types of measured angles, $\theta_{i}$ and $\varphi_{i}$ the bending energies for each DNA conformation were calculated and plotted based on a simple WLC model. The averaged bending energies from the two types of angles are indicated by the dashed lines. The bending energy of the standard DNA model was also calculated and indicated as structure no. 0 as a control.

prediction. (iv) However, after submitting the model to further MD simulations for only $0.1 \mathrm{~ns}$, the calculated energy jumped up to $\sim 80 \%$ of the energy from the EM configuration, thereby confirming that the DNA is more flexible than the WLC prediction. This result suggests that the MD simulations may be the key role to increase the bending energy and result in a more flexible DNA model. (v) To evaluate how the noise in the density map may influence the bending energy, we conducted MD flexible fitting (MDFF) based on the smooth model to constrain its structure with the EM density map. The calculated bending energy immediately jumped up to an even higher level, that is, $\sim 10 \%$ more than that from the EM configurations. This test suggests that the MDFF and noise in the EM density could be critical in causing the DNA model to be more flexible than it should be. (vi) To avoid a potential influence to the energy from the given initial models, a standard and straight model of DNA was used for MD simulations under the same condition, that is, under $0.15 \mathrm{M}$ physiological salt solution, temperature of $298 \mathrm{~K}$ and a pressure of $1 \mathrm{~atm}$. Using NAMD2 for $20 \mathrm{~ns}$ of simulation without any constraint for DNA conformational changes, the equilibration of DNA in a box of $\sim 347.3 \AA \times \sim 93.4 \AA \times \sim 50.8 \AA$ was monitored using the root mean square deviation by visual MD (VMD) ${ }^{31}$ (Supplementary Fig. 14a). The bending energies (Supplementary Fig. 14b) and the distances between the two distal ends (Supplementary Fig. 14c) revealed that the system became nearly balanced after $8 \mathrm{~ns}$. The statistical analyses of the bending energies of the DNA in its last 10 -ns simulations showed that the average energy was $99.1 \pm 10.9 \mathrm{kcal} \mathrm{mol}^{-1}$ with a peak population $(\sim 7.1 \%)$ energy of $97.4 \pm 1.0 \mathrm{kcal} \mathrm{mol}^{-1}$ based on the bending angles of the $\varphi_{i}$ calculation, whereas the average energy was $152.1 \pm 16.1 \mathrm{kcal} \mathrm{mol}^{-1}$ with a peak population $(\sim 4.6 \%)$ energy of $151.3 \pm 1.0 \mathrm{kcal} \mathrm{mol}^{-1}$ based on the bending angles of the $\theta_{i}$ calculation (Supplementary Fig. 14b,d). This energy is surprisingly similar to that of the EM configuration $(\sim 10-15 \%$ lower) (Supplementary Table 2). The statistical analyses of the length between the two distal ends of equilibrated DNA in the last $10 \mathrm{~ns}$ of the simulations showed that the average length was $268.5 \pm 2.1 \AA$ with a peak population $(\sim 18.5 \%)$ length of $267.9 \pm 0.5 \AA$ (Supplementary Fig. 14c,e). The distance is similar to the length at the peak population measured from TEM images. (vii) To evaluate how temperature influences the bending energy measurement using MD simulations, the above processes were repeated under a higher temperature, that is, $310 \mathrm{~K}$ instead of $298 \mathrm{~K}$ (Supplementary Fig. 15). The bending energies calculated under the higher temperature in the last $10 \mathrm{~ns}$ showed that the average energy was $120.8 \pm 14.7 \mathrm{kcal} \mathrm{mol}^{-1}$ with a peak population $(\sim 6.1 \%)$ energy of $115.1 \pm 1.0 \mathrm{kcal} \mathrm{mol}^{-1}$ based on the bending angles of the $\varphi_{i}$ calculation, whereas the average energy was $177.9 \pm 15.6 \mathrm{kcal} \mathrm{mol}^{-1}$ with a peak population $(\sim 5.1 \%)$ energy of $177.5 \pm 1.0 \mathrm{kcal} \mathrm{mol}^{-1}$ based on the bending angles of the $\theta_{i}$ calculation (Supplementary Fig. 15b,d). The energy is increased by $\sim 20 \%$ from those under $298 \mathrm{~K}$ and becomes more similar to that of the EM configuration, suggesting that the temperature is related, but not critical. The statistical analyses on the length showed that the averaged length was $269.5 \pm 2.9 \AA$ with a peak population $(\sim 13.3 \%)$ length of $271.1 \pm 0.5 \AA$ (Supplementary Fig. 15c,e), which is similar to those measured from the EM configurations and $298 \mathrm{~K}$ MD simulation, suggesting that the length is insensitive to the temperature. (viii) To further confirm that the length is insensitive to bending energy, one EM configuration with the DNA length of $\sim 241.0 \AA$ was performed by $\mathrm{MD}$ simulations under a length constrain (Supplementary Fig. 16). This length is close to the mean length of the DNA portion estimated from solution using $\mathrm{SAXS}^{25}$. The bending energy in the last $10 \mathrm{~ns}$ was $105.8 \pm 10.7 \mathrm{kcal} \mathrm{mol}^{-1}$ with a peak population $(\sim 7.3 \%)$ energy of $102.7 \pm 1.0 \mathrm{kcal} \mathrm{mol}^{-1}$ 
based on the bending angles of the $\varphi_{i}$ calculation, whereas the average energy was $163.6 \pm 17.4 \mathrm{kcal} \mathrm{mol}^{-1}$ with a peak population $(\sim 5.0 \%)$ energy of $158.4 \pm 1.0 \mathrm{kcal} \mathrm{mol}^{-1}$ based on the bending angles of the $\theta_{i}$ calculation (Supplementary Fig. 16b,c). This energy is similar to that of the other EM configurations and simulations, suggesting that the length cannot reflect the bending energy and flexibility of DNA.

Above tests showed, although EM density maps contain noise, that the bending energy can be influenced limitedly by the initial models and the EM map configuration. The similar bending energies calculated from different MD simulations and initial models suggested that the DNA is more flexible than WLC prediction. However, we cannot exclude those MD simulations that may result in DNA, which presents more flexibility than WLC prediction.

\section{Discussion}

Although the direct imaging of dsDNA has been previously reported using heavy metal shadowing ${ }^{32,33}$ and NS methods ${ }^{34-36}$, to the best of our knowledge, the 3D structure of an individual dsDNA strand has not previously been achieved. It has been thought that individual dsDNA would be destroyed under the high energy of the electron beam before a $3 \mathrm{D}$ reconstruction, or even a $2 \mathrm{D}$ image, is able to be achieved. Our NS tilt images showing fibre-shaped dsDNA bridging two conjugated nanogold particles demonstrated that the dsDNA can in fact be directly visualized using EM, which is consistent with the recently reported single-molecule DNA sequencing technique via $\mathrm{TEM}^{36}$. The resolutions of our density maps ranged from $\sim 14$ to $\sim 23 \AA$, demonstrating that an intermediate-resolution $3 \mathrm{D}$ structure can be obtained for each individual macromolecule. This capability is consistent with our earlier report of a $\sim 20$ - $\AA$ resolution 3D reconstruction of an individual IgG1 antibody using the same $\operatorname{approach}^{16,17}$.

Notably, a total dose of $\sim 2,000 \mathrm{e}^{-} \AA^{-2}$ used in our ET data acquisition is significantly above the limitation conventionally used in cryo-EM $\left(\sim 80-100 \mathrm{e}^{-} \AA^{-2}\right)$, which can be suspected to have certain artefact from radiation damage. In cryo-EM, the radiation damage could cause sample bubbling, deformation and knockout effects; in NS, only the knockout phenomena is often observed, in which the protein is surrounded by heavy atoms that were kicked out by electron beam. Since the sample was coated with heavy metal atoms and were dried in air, the bubbling and deformation phenomena were not usually observed. The heavy metal atoms that coat the surface of the biomolecule can provide a much higher electron scattering than from a biomolecule only inside lighter atoms. The scattering is sufficiently high to provide enough image contrast at our $120-\mathrm{kV}$ high tension; thus, a further increase to the scattering ability by reducing the high tension to $80 \mathrm{kV}$ may not be necessary for this NS sample. In addition, the heavy atoms can provide more radiation resistance and allow the sample to be imaged under a higher dose condition. The exact dose limitation for NS is still unknown. The radiation damage related artefact in NS samples is knockout, which could reduce the image contrast and lower the tilt image alignment accuracy and $3 \mathrm{D}$ reconstruction resolution. In our study, a total dose of $2,000 \mathrm{e}^{-} \AA^{-2}$ did not cause any obvious knockout phenomena, but provides a sufficiently high contrast for the otherwise barely visible DNA conformations in each tilt series. The direct confirmation of visible DNA in each tilt image is essentially important to us to validate each $3 \mathrm{D}$ reconstruction, especially considering this relatively new approach.

Our 3D reconstruction algorithm used an $a b$ initio real-space reference-projection match iterative algorithm to correct the centres of each tilt images, in which the equal tilt angle step for
$3 \mathrm{D}$ reconstruction of a low contrast and asymmetric macromolecule was used. This method is different from recently reported Fourier-based iterative algorithm, termed equally sloped tomography, in which the pseudo-polar fast Fourier transform, the oversampling method and internal lattice of a targeted nanoparticle are used to achieve $3 \mathrm{D}$ reconstruction at atomic resolution ${ }^{37}$.

It is generally challenging to achieve visualization and $3 \mathrm{D}$ reconstruction on an individual, small and asymmetric macromolecule by other conventional methods; our method demonstrated its capability for $3 \mathrm{D}$ reconstruction of $52 \mathrm{kDa} 84$-bp dsDNA through these studies: IgG1 antibody 3D structural fluctuation $^{17}$, peptide-induced conformational changes on flexible IgG1 antibody ${ }^{5}$, floppy liposome surface binding with $53-\mathrm{kDa}$ proteins $^{38}$, all of which suggest that this method could be used to serve the community as a novel tool for studying flexible macromolecular structures, dynamics and fluctuations of proteins, and for catching the intermediate 3D structure of protein assembling.

DNA-based self-assembling materials have been developed for use in materials science and biomedical research, such as DNA origami designed for targeted drug delivery. The structure, design and control require feedback from the $3 \mathrm{D}$ structure, which could validate the design hypothesis, optimize the synthesis protocol and improve the reproducible capability, while even providing insight into the mechanism of DNA-mediated assembly.

\section{Methods}

Synthesis of DNA-nanogold conjugates. Conjugation of DNA-nanogold was synthesized according to a previously published procedure ${ }^{1}$. In brief, $\sim 5.5-\mathrm{nm}$ nanogold particles were stabilized via exchanging with bis-( $p$-sulfonatophenyl) phenylphosphine. DNA sequences modified with a $5^{\prime}$-thiol moiety were purified via polyacrylamide gel electrophoresis. DNA thiolated at the $5^{\prime}$ end was re-suspended in buffer ( $10 \mathrm{mM}$ Tris pH 8 and $0.5 \mathrm{mM}$ EDTA). Nanogold particles and DNA were combined at a stoichiometric ratio of 1:2 in the presence of a reducing agent. The formed monoconjugates were separated using anion-exchange HPLC, and the fractions were concentrated using an Amicon Ultra spin filter, MW $=100,000$ (EMD Millipore Corp., Billerica, MA, USA). Twenty microlitres of nanogold monoconjugates, each containing complementary strands of DNA, were combined stoichiometrically, as determined by absorption at $520 \mathrm{~nm}$, and were allowed to react overnight at room temperature ${ }^{7}$. The final conjugates were purified from unreacted monoconjugates via agarose gel electrophoresis.
\end{abstract}

\section{Sequences. 84-Base DNA sequence. $5^{\prime}$-thiol-CCGGCGGCCCAGGTGTA TCAGTGTTCGTTGCAAGCTCCAACATCTGAGTACCACGCATACTAT ACTTGAAATATCCGCGCCCGG-3'. \\ 84-Base DNA complement. 5' -thiol-CCGGGCGCGGATATTTCAAGTATA GTATGCGTGGTACTCAGATGTTGGAGCTTGCAACGAACACTGATACAC CTGGGCCGCCGG-3'}

Preparation of cryo-EM and OpNS-EM specimens. The cryo-EM specimens were prepared as described previously ${ }^{39}$. In brief, an aliquot $(\sim 4 \mu \mathrm{l})$ of DNA-nanogold sample at a concentration of $\sim 20 \mu \mathrm{g} \mathrm{ml}^{-1}$ was placed on a glow-discharged lacey carbon film-coated copper grid (Cu-200LC, Pacific Grid-Tech, San Francisco, CA, USA). The samples were blotted with filter paper from both sides at $\sim 90 \%$ humidity and $4{ }^{\circ} \mathrm{C}$ with a Leica EM GP rapid-plunging device (Leica, Buffalo Grove, IL, USA) and then flash-frozen in liquid ethane. The flash-frozen grids were transferred into liquid nitrogen for storage. The NS specimens were prepared by OpNS protocol as described previously ${ }^{19,22}$. In brief, an aliquot $(\sim 4 \mu \mathrm{l})$ of DNA-nanogold sample at a concentration of $\sim 20 \mu \mathrm{g} \mathrm{ml}^{-1}$ was placed on a thin carbon-coated 200-mesh copper grid (Cu-200CN, Pacific Grid-Tech; CF200-Cu, Electron Microscopy Sciences, Hatfield, PA, USA) that had been glow-discharged. After $\sim 1$ min of incubation, the excess solution was blotted with filter paper. The grid was then washed with water and stained with $1 \%(\mathrm{w} / \mathrm{v})$ uranyl formate on Parafilm before air-drying with nitrogen ${ }^{19,22}$.

TEM un-tilted data acquisition and imaging process. Both cryo-EM and OpNS samples were examined using a Zeiss Libra 120 Plus TEM (Carl Zeiss NTS) operating at $120 \mathrm{kV}$ high tension with $20 \mathrm{eV}$ in-column energy filtering at $-178^{\circ} \mathrm{C}$ (for cryo-EM) and room temperature (for NS). A Gatan 915 cryo-holder was used. The micrographs were acquired using a Gatan UltraScan $4 \mathrm{~K} \times 4 \mathrm{~K}$ CCD under a magnification of $20-125 \mathrm{kx}$ (each pixel of the micrographs corresponded to $\sim 0.59$ to $\sim 0.094 \mathrm{~nm}$ in specimens) under a dose of $\sim 5-15 \mathrm{e}^{-} \AA^{-2}$ (for cryo-EM) and 
$\sim 40-90 \mathrm{e}^{-} \AA^{-2}$ (for NS-EM), and defocus of $0.2-3 \mu \mathrm{m}$ (for cryo-EM) and $<1.0 \mu \mathrm{m}$ (for NS-EM). The defocus and astigmatism of each micrograph were examined using EMAN $c t f i t$ software ${ }^{40}$ after the X-ray speckles were removed. The micrographs with distinguishable drift effects were excluded. The remaining micrographs were filtered using a Gaussian boundary high-pass filter within a resolution range of $\sim 200-500 \mathrm{~nm}$.

The statistical analysis of the diameter of the nanogold particles was based on the geometric mean 22 of 1,032 cryo-EM nanogold particles and 606 NS nanogold particles. Each particle was measured based on two perpendicular directions in which one of the diameters was measured along the longest direction of the particle ${ }^{22}$. The DNA lengths were measured by calculating the distance between the centres of two nanogolds (centre-to-centre distance). The lengths of 516 pairs from cryo-EM images and 303 pairs from NS images were measured and then submitted for histogram analyses by using Matlab software.

ET data acquisition and image pre-process. The TEM holder was tilted at angles ranging from $-60^{\circ}$ to $+60^{\circ}$ at $1.5^{\circ}$ increments and controlled using Gatan tomography software that was pre-installed in the microscopes (Zeiss Libra 120 TEM). The TEM was operated under $120 \mathrm{kV}$ with a $20 \mathrm{eV}$ energy filter. The tilt series were acquired using a Gatan Ultrascan $4 \mathrm{~K} \times 4 \mathrm{~K}$ CCD camera under lowdefocus conditions $(<1.0 \mu \mathrm{m})$ and a magnification of $125 \mathrm{kx}(0.094 \mathrm{~nm}$ per pixel). The total electron doses were $\sim 1,500-2,500 \mathrm{e}^{-} \AA^{-2}$. The micrographs were initially aligned together using the IMOD software package ${ }^{41}$. The CTF was then corrected using TOMOCTF ${ }^{42}$. The tilt series of the particles in square windows of $512 \times 512$ pixels $(\sim 48 \mathrm{~nm})$ were semi-automatically tracked and windowed using IPET software ${ }^{10}$

IPET 3D reconstruction. In the IPET reconstruction process ${ }^{10}$, a tilt series of CTF-corrected images containing a single DNA-nanogold particle (in size of $\sim 48 \mathrm{~nm}$ ) was directly back-projected into an ab initio 3D density map as an initial model based on their corresponding goniometer tilt angles. The refinement was started using this initial model to align each tilt image via translational alignment to the projections of the initial model. During the refinement, automatically generated filters and a circular-shaped mask with a Gaussian boundary were sequentially applied to the tilt images and references to increase the alignment accuracy ${ }^{10}$. The resolution was defined based on FSC in which the aligned images were split into two halves based on an odd- or even-numbered index to generate two 3D reconstructions for computing their FSC curve against the spatial frequency shells in Fourier space. The frequency at which the FSC curve first falls to a value of 0.5 was used to represent the resolution of the IPET 3D density map. All of the IPET density maps presented in the figures were low-pass filtered to 16-20 A. The DNA portion SNR in each 3D map was calculated using the equation $\mathrm{SNR}_{y}=\left(I_{\mathrm{s}}-I_{\mathrm{b}}\right) / N_{\mathrm{b}}$, where $I_{s}$ is the average power inside the particle, $I_{b}$ is the average power outside the particle (nanogold area was excluded), and $N_{\mathrm{b}}$ is the s.d. of the noise calculated from the background s.d. outside the particle (nanogold area was excluded). The particle area was defined using a particle-shaped mask generated from the IPET final 3D with low-pass filtering to $\sim 25-30 \AA$ and set as three times its molecular weight. This same method was used to calculate the $2 \mathrm{D}$ SNR. The $2 \mathrm{D}$ mask was generated based on the $3 \mathrm{D}$ projection at each tilt angle.

Conformation of DNA. A standard model of the 84-bp double-helix B-form DNA (dsDNA) was generated using the online DNA sequence to structure tool ${ }^{43}$. By comparing the standard model to the density map, we aligned the best-fit positions onto the EM density map envelope using Chimera ${ }^{44}$. Using the best-fit positions as the target markers, we drove the DNA conformational change of the model to agree with the DNA portion in the 3D density map with a pre-calculated force via a targeted MD (TMD) simulation ${ }^{45}$. After $0.6 \mathrm{~ns}$ of TMD simulation, the DNA was moved onto the density map, and we further equilibrated the DNA conformation in a water solution at room temperature via a MDFF simulation under the constraint of the original density map while protecting the secondary structure. Both TMD and MDFF simulations were performed using NAMD2 software package from the University of Illinois at Urbana-Champaign ${ }^{30}$.

A method to generate a smooth DNA model to match the EM configuration was conducted as follows: the DNA portion between two nanogolds was first marked out by a series of consecutive 3D points. Labelled points were automatically fitted with a smooth quadratic or cubic Bézier curve by GraphiteLifeExplorer software ${ }^{46}$. A DNA model was generated to be as close as possible to the curve with a canonical DNA helix. For comparison, the model was also submitted for refinement by an energy minimization with NAMD2 at $0.15-\mathrm{M}$ salt concentrations. The energy minimization was last for 30,000 steps and followed by 0.1 -ns all-atom simulation. For a further comparison, the model was also constrained by the electron density map using MDFF. All the MD simulations were performed using CHARMM force field ${ }^{26,27}$.

Calculation of dsDNA bending energy. To calculate the DNA bending energy, we measured the local bending angles, as illustrated in Fig. $4 \mathrm{c}-\mathrm{e}$. In detail, the two nearby bases of the standard DNA model were rigidly aligned to the derived dsDNA conformations using $\mathrm{VMD}^{31}$. Based on the centres $\left(C_{i}\right)$ and centre directions (indicated as a vector $\overrightarrow{S_{i}}$ ) of the aligned standard DNA bases, whose orientations are represented by a small cylinder, the two types of DNA bending angles were measured, that is, the angle $\theta_{i}$ formed by the centres of three consecutive cylinders and the angles $\varphi_{i}$ between the central axes of two consecutive cylinders. Therefore, the DNA bend energy $E_{\mathrm{bend}}$ was calculated according to $E_{\text {bend }}=k_{\mathrm{b}} T \frac{l_{\mathrm{p}}}{d} \sum_{i}\left(1-\cos \theta_{i}\right)$ or $E_{\text {bend }}=k_{\mathrm{b}} T \frac{T_{\mathrm{p}}}{d} \sum_{i}\left(1-\cos \varphi_{i}\right)$, where $l_{\mathrm{p}}$ is the dsDNA persistence length $(50 \mathrm{~nm})$ and $d$ is the distance between the base pairs $(3.4 \AA$; ref. 25).

MD simulation of dsDNA bending energy and length in solution. The MD simulation analyses were performed according to the following three steps: (i) a standard model of 84-bp dsDNA was embedded into a cubic box that extended at least $15 \AA$ away from the DNA surface. The box contained a total of 49,679 TIP3P water molecules, $307 \mathrm{Na}^{+}$atoms and $141 \mathrm{Cl}^{-}$atoms to simulate the $0.15-\mathrm{M}$ physiological salt concentration and was constructed using $\mathrm{VMD}^{31}$. The system, which contained a total of 154,813 atoms, was subjected to energy minimization via 20,000 steps to remove the atomic clashes using the NAMD2. The DNA backbone atoms were fixed in the first 10,000 steps. In the second 10,000 steps, the DNA backbone atoms were constrained under a force constant of $5 \mathrm{kcal} \mathrm{mol}^{-1} \AA^{-2}$. The energy-minimized system was subsequently heated from 0 to $298 \mathrm{~K}$ over $120 \mathrm{ps}$ to initiate the 20-ns all-atom MD simulations. The systems under $1 \mathrm{~atm}$ and $298 \mathrm{~K}$ reached equilibration after $10 \mathrm{~ns}$; the DNA backbone constraints were removed after the first $0.2 \mathrm{~ns}$ of the simulations. During the simulations, the temperature was maintained via Langevin dynamics ${ }^{47}$ with a damping coefficient of $5 \mathrm{ps}$, and the $1 \mathrm{~atm}$ pressure was maintained using the Langevin piston Nose-Hoover method ${ }^{48}$ with a piston period of $100 \mathrm{fs}$ and a decay time of $50 \mathrm{fs}$. Periodic boundary conditions and a cutoff distance of $12 \AA$ for van der Waals' interactions were applied, and the Particle-Mesh Ewald method ${ }^{49}$ with a grid spacing of $<1 \AA$ was used to compute the long-range electrostatic interactions. (ii) The above system was submitted again under a higher balanced temperature of $310 \mathrm{~K}$ instead of $298 \mathrm{~K}$. (iii) The 11th dsDNA conformation derived from the previous flexible docking was embedded into a cubic box containing 52,093 TIP3P water molecules, $313 \mathrm{Na}^{+}$atoms and $147 \mathrm{Cl}^{-}$atoms. The criterion to select this conformation as a representative conformation of the dsDNA model was a length of $\sim 241.0 \AA$, which was close to the mean length of the DNA estimated from solution by SAXS ${ }^{25}$. The system, which contained 162,067 atoms, was subjected to energy minimization via 10,000 steps to remove the atomic clashes using NAMD2. The energy-minimized system was subsequently heated from 0 to $293 \mathrm{~K}$ over $60 \mathrm{ps}$ to initiate the 20-ns all-atom MD simulation, in which the end-to-end distance of this DNA was constrained at $241.0 \AA$ under a force constant of $50 \mathrm{kcal} \mathrm{mol}^{-1} \AA^{-2}$. This system under $1 \mathrm{~atm}$ and $293 \mathrm{~K}$ reached equilibration after $10 \mathrm{~ns}$ of simulation. In the above processes, the bending energies and length of the dsDNA conformations in the last $10 \mathrm{~ns}$ were submitted to the statistical analyses.

\section{References}

1. Alivisatos, A. P. et al. Organization of 'nanocrystal molecules' using DNA. Nature 382, 609-611 (1996).

2. Mirkin, C. A., Letsinger, R. L., Mucic, R. C. \& Storhoff, J. J. A DNA-based method for rationally assembling nanoparticles into macroscopic materials. Nature 382, 607-609 (1996).

3. Elghanian, R., Storhoff, J. J., Mucic, R. C., Letsinger, R. L. \& Mirkin, C. A. Selective colorimetric detection of polynucleotides based on the distancedependent optical properties of gold nanoparticles. Science 277, 1078-1081 (1997).

4. Wu, X. et al. Immunofluorescent labeling of cancer marker Her2 and other cellular targets with semiconductor quantum dots. Nat. Biotechnol. 21, 41-46 (2003).

5. Zheng, J. et al. Two-dimensional nanoparticle arrays show the organizational power of robust DNA motifs. Nano Lett. 6, 1502-1504 (2006).

6. Barrow, S. J., Wei, X., Baldauf, J. S., Funston, A. M. \& Mulvaney, P. The surface plasmon modes of self-assembled gold nanocrystals. Nat. Commun. 3, 1275 (2012).

7. Claridge, S. A., Liang, H. W., Basu, S. R., Frechet, J. M. \& Alivisatos, A. P. Isolation of discrete nanoparticle-DNA conjugates for plasmonic applications. Nano Lett. 8, 1202-1206 (2008).

8. Jones, M. R., Seeman, N. C. \& Mirkin, C. A. Nanomaterials. Programmable materials and the nature of the DNA bond. Science 347, 1260901 (2015).

9. Liao, M., Cao, E., Julius, D. \& Cheng, Y. Structure of the TRPV1 ion channel determined by electron cryo-microscopy. Nature 504, 107-112 (2013).

10. Zhang, L. \& Ren, G. IPET and FETR: experimental approach for studying molecular structure dynamics by cryo-electron tomography of a singlemolecule structure. PloS ONE 7, e30249 (2012).

11. Milne, J. L. \& Subramaniam, S. Cryo-electron tomography of bacteria: progress, challenges and future prospects. Nat. Rev. Microbiol. 7, 666-675 (2009).

12. Komeili, A., Li, Z., Newman, D. K. \& Jensen, G. J. Magnetosomes are cell membrane invaginations organized by the actin-like protein MamK. Science 311, 242-245 (2006).

13. Hoppe, W., Gassmann, J., Hunsmann, N., Schramm, H. J. \& Sturm, M. Three dimensional reconstruction of individual negatively stained yeast fatty-acid 
synthetase molecules from tilt series in the electron microscope. Hoppe-Seylers Z Physiol Chem 355, 1483-1487 (1974).

14. Frank, J. Electron Tomography, Methods for Three-Dimensional Visualization of Structures in the Cell (Springer, 2006).

15. Unwin, P. N. \& Henderson, R. Molecular structure determination by electron microscopy of unstained crystalline specimens. J. Mol. Biol. 94, 425-440 (1975).

16. Tong, H. et al. Peptide-conjugation induced conformational changes in human IgG1 observed by optimized negative-staining and individual-particle electron tomography. Sci. Rep. 3, 1089 (2013).

17. Zhang, X. et al. 3D structural fluctuation of IgG1 antibody revealed by individual particle electron tomography. Sci. Rep. 5, 9803 (2015).

18. Lu, Z. et al. Calsyntenin-3 molecular architecture and interaction with neurexin 1alpha. J. Biol. Chem. 289, 34530-34542 (2014).

19. Zhang, L. et al. An optimized negative-staining protocol of electron microscopy for apoE4 POPC lipoprotein. J. Lipid Res. 51, 1228-1236 (2010).

20. Rames, M., Yu, Y. \& Ren, G. Optimized negative staining: a high-throughput protocol for examining small and asymmetric protein structure by electron microscopy. J. Vis. Exp. 90, e51087 (2014).

21. Ohi, M., Li, Y., Cheng, Y. \& Walz, T. Negative staining and image classification - powerful tools in modern electron microscopy. Biol. Proced. Online 6, 23-34 (2004).

22. Zhang, L. et al. Morphology and structure of lipoproteins revealed by an optimized negative-staining protocol of electron microscopy. J. Lipid Res. 52, 175-184 (2011).

23. Zhang, L. et al. Structural basis of transfer between lipoproteins by cholesteryl ester transfer protein. Nat. Chem. Biol. 8, 342-349 (2012).

24. Chen, Q. et al. 3D motion of DNA-Au nanoconjugates in graphene liquid cell electron microscopy. Nano Lett. 13, 4556-4561 (2013).

25. Mastroianni, A. J., Sivak, D. A., Geissler, P. L. \& Alivisatos, A. P. Probing the conformational distributions of subpersistence length DNA. Biophys. J. 97, 1408-1417 (2009).

26. Foloppe, N. \& MacKerell, A. D. All-atom empirical force field for nucleic acids: I. Parameter optimization based on small molecule and condensed phase macromolecular target data. J. Comput. Chem. 21, 86-104 (2000).

27. MacKerell, A. D. \& Banavali, N. K. All-atom empirical force field for nucleic acids: II. Application to molecular dynamics simulations of DNA and RNA in solution. J. Comput. Chem. 21, 105-120 (2000).

28. Bustamante, C., Smith, S. B., Liphardt, J. \& Smith, D. Single-molecule studies of DNA mechanics. Curr. Opin. Struct. Biol. 10, 279-285 (2000).

29. Bustamante, C., Bryant, Z. \& Smith, S. B. Ten years of tension: single-molecule DNA mechanics. Nature 421, 423-427 (2003).

30. Kale, L. et al. NAMD2: Greater scalability for parallel molecular dynamics. J. Comput. Phys. 151, 283-312 (1999).

31. Humphrey, W., Dalke, A. \& Schulten, K. VMD: visual molecular dynamics. J. Mol. Graph. 14, 33-38 (1996).

32. Hall, C. E. Method for the observation of macromolecules with the electron microscope illustrated with micrographs of DNA. J. Biophys. Biochem. Cytol. 2, 625-628 (1956)

33. Beer, M. Electron microscopy of unbroken DNA molecules. J. Mol. Biol. 3, 263-266 (1961)

34. Zobel, C. R. \& Beer, M. Electron stains. I. Chemical studies on the interaction of DNA with uranyl salts. J. Biophys. Biochem. Cytol. 10, 335-346 (1961).

35. Beer, M. \& Zobel, C. R. Electron stains. II: Electron microscopic studies on the visibility of stained DNA molecules. J. Mol. Biol. 3, 717-726 (1961).

36. Bell, D. C. et al. DNA base identification by electron microscopy. Microsc. Microanal. 18, 1049-1053 (2012).

37. Scott, M. C. et al. Electron tomography at 2.4-angstrom resolution. Nature 483, 444-447 (2012).

38. Zhang, M. et al. HDL surface lipids mediate CETP binding as revealed by electron microscopy and molecular dynamics simulation. Sci. Rep. 5, 8741 (2015).

39. Jones, M. K. et al. Assessment of the validity of the double superhelix model for reconstituted high density lipoproteins: a combined computationalexperimental approach. J. Biol. Chem. 285, 41161-41171 (2010).

40. Ludtke, S. J., Baldwin, P. R. \& Chiu, W. EMAN: semiautomated software for high-resolution single-particle reconstructions. J. Struct. Biol. 128, 82-97 (1999).

41. Kremer, J. R., Mastronarde, D. N. \& McIntosh, J. R. Computer visualization of three-dimensional image data using IMOD. J. Struct. Biol. 116, 71-76 (1996).
42. Fernandez, J. J., Li, S. \& Crowther, R. A. CTF determination and correction in electron cryotomography. Ultramicroscopy 106, 587-596 (2006).

43. Arnott, S., Campbell-Smith, P. J. \& Chandrasekaran, R. In Handbook of Biochemistry and Molecular Biology, Vol II (ed. Fasman G.P.) 3rd edn, 411-422 (CRC Press, Cleveland, 1976).

44. Pettersen, E. F. et al. UCSF Chimera-a visualization system for exploratory research and analysis. J. Comput. Chem. 25, 1605-1612 (2004).

45. Schlitter, J., Engels, M. \& Kruger, P. Targeted molecular dynamics: a new approach for searching pathways of conformational transitions. J. Mol. Graph. 12, 84-89 (1994)

46. Hornus, S., Levy, B., Lariviere, D. \& Fourmentin, E. Easy DNA modeling and more with GraphiteLifeExplorer. PLoS ONE 8, e53609 (2013).

47. Grest, G. S. \& Kremer, K. Molecular dynamics simulation for polymers in the presence of a heat bath. Phys. Rev. A 33, 3628-3631 (1986).

48. Feller, S. E., Zhang, Y. H., Pastor, R. W. \& Brooks, B. R. Constant-pressure molecular-dynamics simulation - the Langevin Piston method. J. Chem. Phys. 103, 4613-4621 (1995)

49. Darden, T., York, D. \& Pedersen, L. Particle mesh Ewald - an N.Log(N) method for Ewald sums in large systems. J. Chem. Phys. 98, 10089-10092 (1993).

\section{Acknowledgements}

We thank Drs Qian Chen and Phillip Geissler for their discussion and comments, and Mr Matthew Rames for editing. This material is based upon work supported by the National Science Foundation under grant DMR-1344290. Work at the Molecular Foundry was supported by the Office of Science, Office of Basic Energy Sciences of the US Department of Energy under contract no. DE-AC02-05CH11231. G.R. is supported by the National Heart, Lung, and Blood Institute of the National Institutes of Health (no. R01HL115153) and the National Institute of General Medical Sciences of the National Institutes of Health (no. R01GM104427). L.Z. is partly supported by National Natural Science Foundation of China (no. 11504287) and Young Talent Support Plan of Xi'an Jiaotong University; Z.L. and J.L. are partly supported by the National Basic Research Program of Ministry of Science and Technology, China (no. 2015CB553602).

\section{Author contributions}

This project was initiated and designed by J.M.S., P.A. and G.R., and refined by L.Z. and G.R. J.S. prepared the conjugates. L.Z., H.T., Z.L. and G.R. prepared TEM samples and/or acquired the data. L.Z. and G.R. processed the data, and L.Z. solved the IPET 3D structures. X.Z., M.Z. and L.Z. docked the model, and D.L. measured the angles and computed/analysed the energies of dsDNA in solution by MD simulations. L.Z., J.M.S., D.L., P.A., J.L. and G.R. interpreted and manipulated the structures. G.R. drafted the initial manuscript, which was revised by L.Z., J.M.S., X.Z., D.L., Z.L., H.T., M.Z., J.L. and P.A.

\section{Additional information}

Accession codes: TEM 3D density maps of 14 DNA-nanogold conjugates are available from the EM data bank as EMDB IDs 2948-2961.

Supplementary Information accompanies this paper at http://www.nature.com/ naturecommunications

Competing financial interests: The authors declare no competing financial interests.

Reprints and permission information is available online at http://npg.nature.com/ reprintsandpermissions/

How to cite this article: Zhang, L. et al. Three-dimensional structural dynamics and fluctuations of DNA-nanogold conjugates by individual-particle electron tomography. Nat. Commun. 7:11083 doi: 10.1038/ncomms11083 (2016).

This work is licensed under a Creative Commons Attribution 4.0 International License. The images or other third party material in this article are included in the article's Creative Commons license, unless indicated otherwise in the credit line; if the material is not included under the Creative Commons license, users will need to obtain permission from the license holder to reproduce the material. To view a copy of this license, visit http://creativecommons.org/licenses/by/4.0/ 